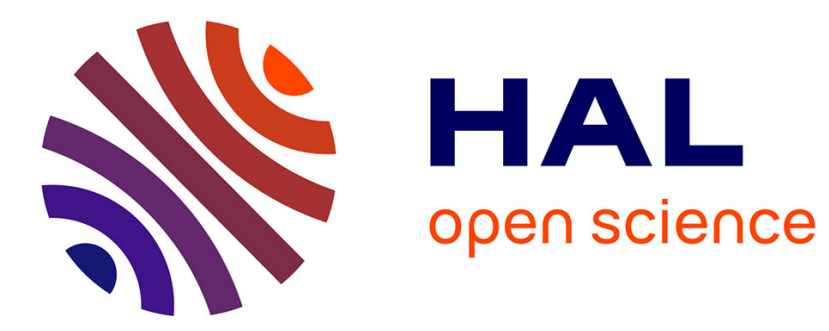

\title{
Foam invasion through a single pore
}

\author{
Aline Delbos, Olivier Pitois
}

\section{To cite this version:}

Aline Delbos, Olivier Pitois. Foam invasion through a single pore. Physical Review E: Statistical, Nonlinear, and Soft Matter Physics, 2011, 84 (1), pp.011404. 10.1103/PhysRevE.84.011404 . hal00790369

\section{HAL Id: hal-00790369 \\ https://hal.science/hal-00790369}

Submitted on 1 Sep 2015

HAL is a multi-disciplinary open access archive for the deposit and dissemination of scientific research documents, whether they are published or not. The documents may come from teaching and research institutions in France or abroad, or from public or private research centers.
L'archive ouverte pluridisciplinaire $\mathbf{H A L}$, est destinée au dépôt et à la diffusion de documents scientifiques de niveau recherche, publiés ou non, émanant des établissements d'enseignement et de recherche français ou étrangers, des laboratoires publics ou privés. 


\title{
Foam invasion through a single pore
}

\author{
Aline Delbos* and Olivier Pitois \\ Université Paris-Est, Laboratoire de Physique des Matériaux Divisés et des Interfaces, CNRS FRE 3300, 5 boulevard Descartes, \\ F-77454 Marne la Vallée Cedex 2, France
}

(Received 24 February 2011; published 27 July 2011)

\begin{abstract}
We investigate experimentally the behavior of liquid foams pumped at a given flow rate through a single pore, in the situation where the pore diameter is smaller than the bubble diameter. Results reveal that foam invasion can be observed only within a restricted range of values for the dimensionless flow rate and the foam liquid fraction. Within this foam invasion regime, the liquid content of invading foams is measured to be three times higher than the initial liquid content. Outside this regime, both gas alone and liquid alone invasion regimes can be observed. The gas invasion regime results from the rupture of foam films during local T1, during bubble rearrangements events induced by foam flow, whereas the liquid invasion regime is allowed by the formation of a stable cluster of jammed bubbles at the pore's opening.
\end{abstract}

DOI: 10.1103/PhysRevE.84.011404

PACS number(s): 82.70.Rr, 47.55.dk, 83.60.La

\section{INTRODUCTION}

Foam is a gas phase dispersed within a liquid phase and stabilized by a surfactant adsorbed at the gas-liquid interface. It has been recognized as a complex fluid with remarkable rheological properties [1], undergoing plastic flow under high applied stress, but behaving as an elastic solid under lower stress. This rich mechanical behavior has motivated numerous applications. Furthermore, its dispersed nature gives it unique properties to profoundly affect the flow patterns of fluids within porous media. It is advantageously used for enhanced oil recovery, so that the scope of research in this area has rapidly expanded [2], including the flow of confined foams and local pore-scale mechanisms for foam lamellae creation or destruction [3]. Other studies have focused on velocity field and bubble elongation, as quasi-two-dimensional dry foam [4-6] or wet foam [7] is flowing through a constriction. In these experimental studies, the constriction is used to establish and test rheological models for complex fluids.

In this paper, we investigate the invasion process of foam through a single cylindrical pore. In the present situation, the pore aperture is smaller than the bubble size and foam drainage is controlled. In contrast to previous studies, we show that there exist conditions for which a stable bubble cluster can form at the pore's opening, preventing foam flow though the pore. As the liquid can permeate through the bubble cluster, we report very large fluctuations for the ratio of fluid phases entering the pore.

\section{EXPERIMENTS}

A monodisperse foam is generated in a column by bubbling diazote in a solution of tetradecyltrimethylammonium bromide (TTAB, from Sigma-Aldrich) concentrated at $3 \mathrm{~g} / 1$ in pure water. The bulk viscosity, density and surface tension of the solution are, respectively, $\eta=10^{-3} \mathrm{~Pa} \mathrm{~s}, \rho=10^{3} \mathrm{~kg} / \mathrm{m}^{3}$, and $\gamma=37 \mathrm{mN} / \mathrm{m}$. The size of the column depends on the bubble size; it is chosen in such a way that the ratio of the

\footnotetext{
*Present address: University of Massachusetts Amherst, Department of Polymer Science and Engineering, 120 Governors Drive, Amherst, Massachusetts 01003, USA; adelbos@ polysci.umass.edu
}

column diameter over the bubble diameter is $>20$. To measure the size of the bubbles we sample tens of bubbles, squeeze them between two glass plates separated by $100 \mu \mathrm{m}$, and measure the surface exposed with a microscope. Using volume conservation, we calculate the average bubble volume and the bubble radius $R$. For this study, bubbles with radii ranging from 0.3 to $1.3 \mathrm{~mm}$ have been produced with a needle. The standard deviation of the bubble distribution was measured to be $<4 \%$.

An entry point along the column wall (at midheight) is used to introduce a thin glass capillary into the foam sample and to pump up the foam (see Fig. 1). The capillary is connected to a syringe pump, allowing for the volumetric flow rate, $q$, to be imposed through the capillary. The capillary length is $12.5 \mathrm{~cm}$, and its radius $r_{t}$ ranges between 0.145 and $0.70 \mathrm{~mm}$ (RingCaps). Images of the glass capillary are recorded during the experiments and are used to analyze the sizes of invading bubbles. The liquid content of invading foams is measured by weighing the capillary before and after the experiment. With this method, the liquid fraction is measured with a precision close to $5 \times 10^{-4}$.

During the experiment, the upper part of the foam is wetted by a constant liquid flow rate $Q$, or equivalently a liquid flux $v_{d}=Q / S$, where $S$ is the column cross section. Under these steady drainage conditions, the foam liquid fraction $\varepsilon$, is uniform throughout the whole sample and it will be referred to as $\varepsilon_{\infty}$, whose value is related to $v_{d}$ through the foam permeability $K$. Introducing the characteristic liquid velocity $V=\rho g R^{2} / \eta$ and the dimensionless foam permeability $\tilde{K}=$ $K / R^{2}$, one can write

$$
v_{d}=\tilde{K}\left(\varepsilon_{\infty}\right) V \quad \text { or } \quad \varepsilon_{\infty}=\tilde{K}^{-1}\left(v_{d} / V\right),
$$

where $\tilde{K}^{-1}$ is the inverse function of $\tilde{K}$. The latter has been already determined for the foaming solution used in this work and is published elsewhere [8]:

$$
\tilde{K}=2.68 \times 10^{-3} \varepsilon_{\infty}^{3 / 2} .
$$

Thus, in this experiment, tuning $v_{d}$ allows for the liquid fraction to be controlled throughout the foam column, within a range $0.005-0.1$. We follow a slightly different procedure to reach very small liquid fractions: $10^{-4}<\varepsilon_{\infty}<5 \times 10^{-3}$. Once 


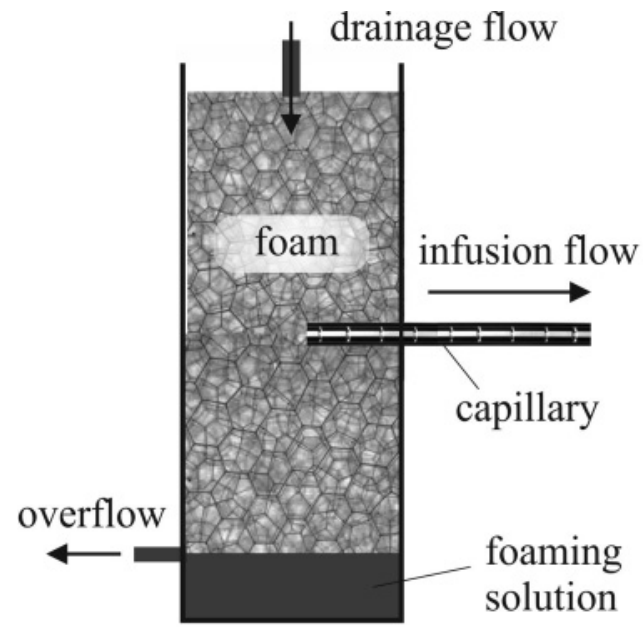

FIG. 1. Experimental setup. The column containing the foam is wetted from the top in order to control liquid drainage and foam liquid fraction. Fluids are forced to invade the pore (capillary) by imposing an infusion flow rate through the pore. Generally, foam invades the pore, but as explained in this paper, there exist conditions for which invading fluid is liquid only, or instead gas only.

the foam has filled the tube, it is allowed to drain freely, so that at the height of the capillary, the liquid fraction $\varepsilon(t)$ slowly decreases as a function of time. $\varepsilon(t)$ is followed in measuring the evolution for the bubbles' radii of curvature at the column wall [9]. Then, experiments are performed when $\varepsilon(t)$ reaches the desired value. For these very small liquid fractions, we were not able to measure the liquid content in the capillary so that we focused on the images of the capillary in order to identify the ratio of gas and liquid passing through the pore.

Further experiments have been done in order to grab images of the capillary's opening during invasion. In that case, the capillary was deeply inserted in the foam column, so that the capillary's opening was close to the opposite wall from the entry point.

\section{RESULTS}

Measurements for the liquid fraction of invading foams $\varepsilon_{t}$ have been performed for several values of bubble radius $R$, foam liquid fraction $\varepsilon_{\infty}$, pore radius $r_{t}$, and infusion rate $q$. An example of the effect of $q$ is presented in Fig. 2 for two foam liquid fractions. At high infusion rates, foams invade the pore, whatever the value of $\varepsilon_{\infty}$. Values measured for $\varepsilon_{t}$ appear to be significantly larger than $\varepsilon_{\infty}$, indicating that a proportion of draining liquid enters the pore during the foam invasion process. For the smallest foam liquid fraction, $\varepsilon_{t}$ does not depend upon the infusion rate over three orders of magnitude. On the other hand, for the largest foam liquid fraction, a sharp transition is observed as the infusion rate is decreased, resulting in a pure liquid invasion regime. A critical value $q^{*}$ can be defined as the infusion rate for which the transition between the foam invasion regime and the liquid invasion regime is observed. As shown in the following, $q^{*}$ depends on $R, r_{t}$, and $\varepsilon_{\infty}$. Images of the pore have been grabbed in the liquid invasion regime and reveal that foam bubbles organize

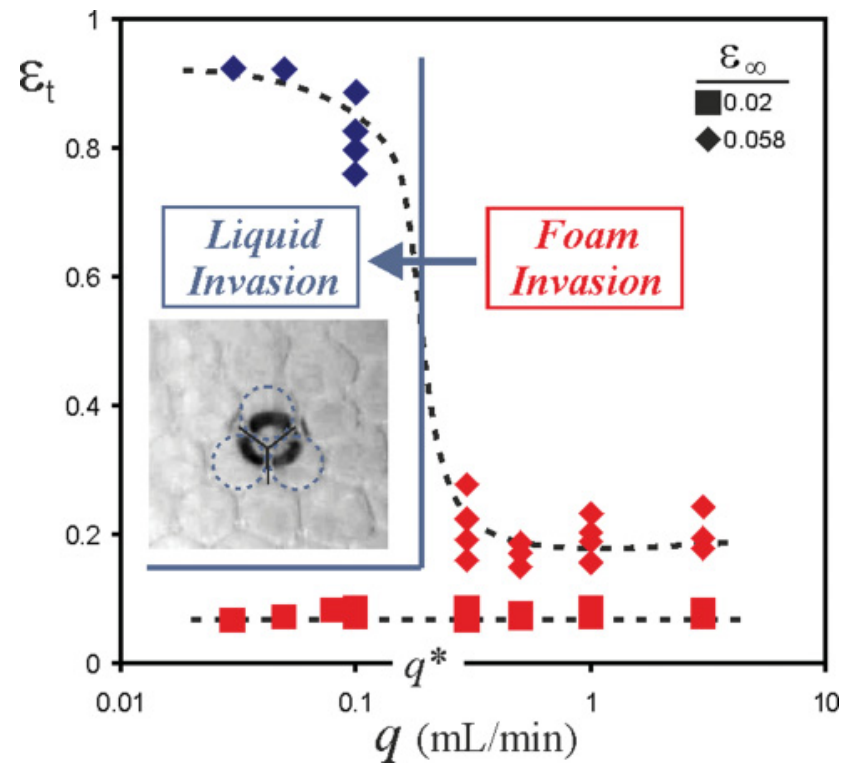

FIG. 2. (Color online) Liquid volume fraction of fluids invading the pore as a function of infusion flow rate, for two foam liquid fractions $\left(\varepsilon_{\infty}\right)$. The foam bubbles and the pore are, respectively, $1 \mathrm{~mm}$ and $0.5 \mathrm{~mm}$ in radius. For the largest foam liquid fraction, a marked transition is observed for a given value $q^{*}$ of the infusion flow rate. For $q<q^{*}$, a stable bubble cluster is formed at the pore's opening and prevents foam bubbles from invading the pore (see the image showing three symmetrically organized bubbles). This invasion regime is referred to as the liquid invasion regime.

at the pore's opening: A cluster of jammed bubbles forms and support the pumping pressure. The cluster prevents foam bubbles from invading the pore but allows permeation of the

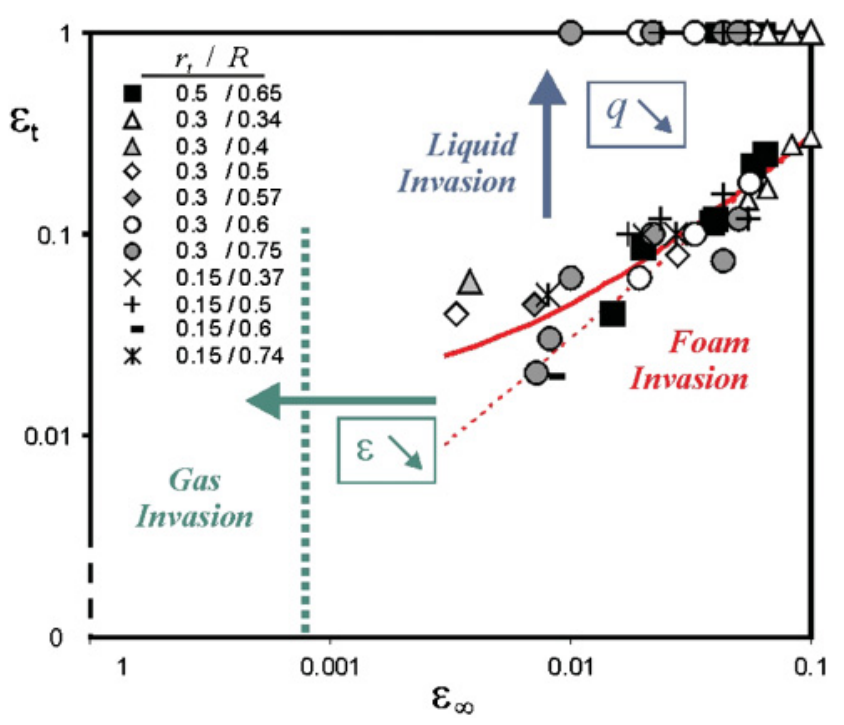

FIG. 3. (Color online) Diagram showing the liquid volume fraction of fluids invading the pore as a function of foam liquid fraction, for several values of bubble radius $(R)$ and pore aperture $\left(r_{t}\right)$. The dotted line shows the relation $\varepsilon_{t}=3 \varepsilon_{\infty}$; the solid line shows the relation $\varepsilon_{t}=3 \varepsilon_{\infty}+b \varepsilon_{t}^{+}$(see text for more details). Arrows indicate transitions for the foam invasion regime towards the liquid invasion regime (controlled by the infusion flow rate) and the gas invasion regime (controlled by the foam liquid fraction). 


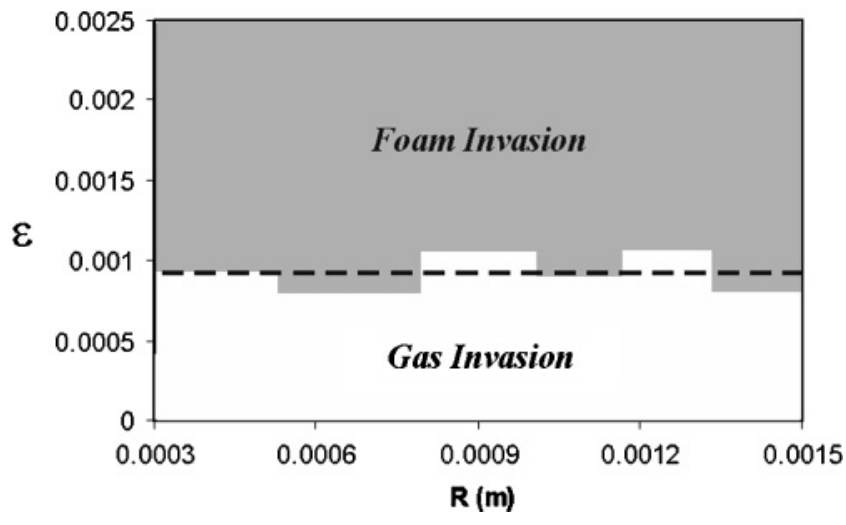

FIG. 4. The diagram shows observed transition behavior of foam when forced to invade a pore, as a function of foam liquid fraction $(\varepsilon)$ and bubble radius $(R)$. Foam is found to invade the pore for liquid fractions above $\sim 0.001$, whatever the bubble size. For lower liquid fractions, only the gas phase invades the pore.

liquid. Note that one or two bubbles generally enter the pore before the stable liquid invasion regime begins. This artifact accounts for the values $\varepsilon_{t}<1$ presented in Fig. 2. Note also that images obtained in the foam invasion regime have not provided evidence for such a bubble ordering at the pore's opening.

The liquid fraction of invading foams is plotted as a function of $\varepsilon_{\infty}$ in Fig. 3. Observed transitions towards the liquid invasion regime are indicated. In the foam invasion regime, $\varepsilon_{t}$ is shown to decrease as $\varepsilon_{\infty}$ decreases, whatever the bubble size and the pore size, but in any case, $\varepsilon_{t}>\varepsilon_{\infty}$. At very low liquid fractions $\left(10^{-4}<\varepsilon_{\infty}<5 \times 10^{-3}\right)$ we observed another transition in the invasion behavior: Below a critical value for the liquid fraction, that will be referred to as $\varepsilon_{\text {crit }}$, only gas enters the pore. As presented in Fig. 4, this transition was found not to depend on $r_{t}, q$, or $R$, and the average value for $\varepsilon_{\text {crit }}$ was measured to be close to $8 \times 10^{-4}$. Images of the foam area close to the pore's opening have revealed foam film rupture events during the infusion process. As a result, bubbles coalesce before they enter the pore. Generally, a large gas cavity is formed at the pore's opening and is sucked into the pore during the infusion process. Further coalescence events with neighboring bubbles make this cavity grow and prevent liquid from being connected with the pore. The introduction of some amount of liquid in the pore space was found to be marginal. Sometimes-especially with foams made of large bubbles-coalescence events occur simultaneously over large foam areas, causing the collapse of the foam around the pore's opening. Note that for such situations, foam collapse has been clearly attributed to the infusion process.

Finally, we noticed that the size of invading gas bubbles in the foam invasion regime is generally equal to that of the foam bubbles, i.e., there is generally no bubble fractionation during the foam invasion process, whatever the infusion rate. This appeared to be true for ratios $R / r_{t}<6$, but for larger values, i.e., the largest foam bubbles and smallest pore size, fractionation is, however, observed. Images of the pore within the conditions $R / r_{t} \geqslant 6$ are presented in Fig. 5 and bring some insight into the fractionation mechanism. It is seen that foam bubbles press against each other and exchange their place in front of the pore's opening. When a bubble is sucked into the pore, the volume it occupies in the foam shrinks and this induces a topological rearrangement with its neighbors, so that another foam bubble moves in front of the pore. In other words, the fractionation seems to result from local bubble rearrangement close to the pore's opening.

\section{DISCUSSION}

\section{A. Liquid invasion regime}

In this experimental configuration, the suction flow rate is imposed and the resulting pressure at the pore entrance drives the liquid. Note, however, that this pressure acts on both liquid and gas bubbles, so that there exists a critical pressure value below which liquid can enter alone, and above which bubbles can enter with liquid. The liquid invasion regime is thus limited by the capillary pressure required to force one bubble to enter the pore. A classical way for estimating this critical pressure is to write: $P=P_{b}-2 \gamma / r_{t}$, where $P_{b}$ is the gas bubble pressure. However, images have revealed that foam bubbles jam at the pore's opening, so that the effective pore aperture is reduced due to the presence of other bubbles forming the cluster. The bubble entry is thus expected to occur when the magnitude of the driving pressure allows for a radius of curvature smaller than $r_{t}$ to be imposed on the bubble surface, $r^{*}=r_{t} / a$, where $a$ is a constant larger than unity. The simplest way for estimating $a$ is to consider that three bubbles are symmetrically positioned around the pore's opening, so

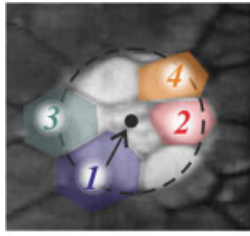

(a)

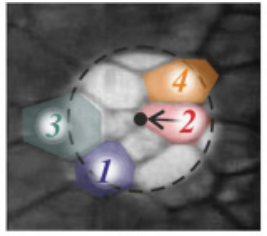

(b)

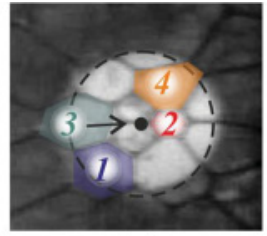

(c)

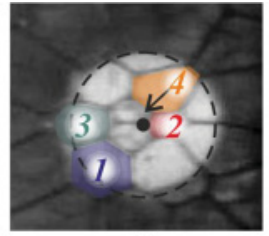

(d)

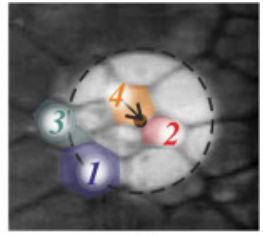

(e)

FIG. 5. (Color online) Illustration of the bubble fractionation mechanism during foam invasion. The pore's opening cannot be distinguished and it as been marked with a solid black circle; the dotted circle shows the external side of the pore (5-mm diam). Numbers are used to mark bubbles around the pore's opening. (a) Bubble 1 is invading the pore (note that the bubble-pore connection is covered by two small bubbles). (b) Bubble 2 invades the pore before bubble 1 has completely invaded the pore, thus highlighting the bubble fractionation mechanism. A topological rearrangement occurred between (b) and (c), so that bubble 3 is now connected to the pore. (d) Bubble 4 is connected to the pore and experiences a new topological rearrangement between (d) and (e). None of the bubbles was allowed to invade the pore without fractionation. 


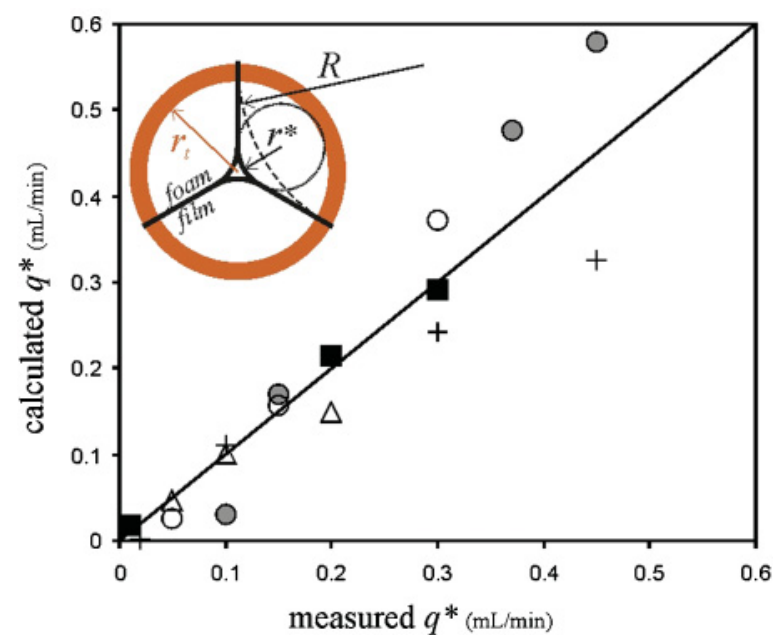

FIG. 6. (Color online) Comparison of measured values for the infusion flow rate corresponding to the transition between the foam invasion regime and the liquid invasion regime, with theoretical values given by Eq. (7), using $a=2.26$. The sketch shows the curvatures of three contacting bubbles (radius $R$ ) jammed at the pore's opening (aperture radius $r_{t}$ ). As the local liquid fraction is decreased in front of the pore's opening, contacting areas (foam films) increase, and the smallest radius of curvature of the bubbles decreases. When this radius is decreased to $r^{*}$, one of the three bubbles can invade the pore in passing through the gap between the pore wall and the two other contacting bubbles.

that $a$ is geometrically defined by $a=2+(2 / \sqrt{3}-1) \approx 2.16$ (see the sketch presented in Fig. 6). The magnitude of the driving pressure also sets the foam liquid fraction for the bubble cluster, $\varepsilon^{*}$, which can be estimated using the following relation: $\varepsilon \approx r^{2} / 3 R^{2}$ [10,11], where $r$ is the smallest radius of curvature of the bubbles' surface. Thus, the criterion for bubble invasion can be expressed in terms of liquid fraction for the bubble cluster:

$$
\varepsilon^{*}=\frac{1}{3 a^{2}}\left(\frac{r_{t}}{R}\right)^{2} .
$$

Now, one has to relate the flow rate of the invading liquid to $\varepsilon^{*}$. As a stating point, one writes Darcy's law along the radial coordinate $\ell$ ( $\ell=0$ at the pore's opening):

$$
\frac{q}{4 \pi \ell^{2}}=\frac{K_{\ell}}{\eta} \frac{d P_{\ell}}{d \ell}
$$

where $K_{\ell}$ and $P_{\ell}$ are, respectively, the permeability and the liquid pressure at $\ell$. $P_{\ell}$ is related to $P_{b}$ and to $r$ through the Laplace law. For liquid foam, this can be expressed as a function of the bubble size and the local liquid fraction $\left(\varepsilon_{\ell}\right)$ by $P_{\ell} \simeq P_{b}-\gamma \varepsilon_{\ell}^{1 / 2} / \sqrt{3} R[10,11]$, so that the pressure gradient becomes

$$
\frac{d P_{\ell}}{d \ell}=\frac{\gamma}{2 \sqrt{3} R} \varepsilon_{\ell}^{-3 / 2} \frac{d \varepsilon_{\ell}}{d \ell}
$$

Using Eqs (2) and (5), Eq. (4) becomes

$$
\ell^{2} \frac{d \varepsilon_{\ell}}{d \ell}=C_{1} \frac{q \eta}{R \gamma}
$$

with $C_{1} \approx 85$. Integration of (6), assuming that far from the pore's opening $(\ell \approx+\infty) \varepsilon_{\ell}=\varepsilon_{\infty}$, and that $\varepsilon_{\ell}=\varepsilon^{*}$ at $\ell=R$ (bubble cluster), provides the following expression for the infusion flow rate at the transition between the liquid invasion regime and the foam invasion regime:

$$
q^{*}=\frac{1}{C_{1}} \frac{\gamma}{\eta} R^{2}\left[\varepsilon_{\infty}-\frac{1}{3 a^{2}}\left(\frac{r_{t}}{R}\right)^{2}\right] .
$$

Experimental results are now compared with theoretical values provided by Eq. (7) for $q^{*}$, in treating the constant $a$ as a fit parameter (see Fig. 6). A reasonable agreement is obtained using $a=2.26$, which is close to the expected value of 2.16.

Note also that it could be expected that decreasing the infusion flow rate to extremely low values would allow for the liquid invasion regime to be observed, whatever the foam liquid fraction. However, one has to consider that for extremely small flow rates, foam ripening (the exchange of gas between bubbles) becomes significant and this induces bubble rearrangements. In such a situation, the bubble cluster is continuously destroyed by topological rearrangements and the liquid invasion regime is not observed, all the more that the characteristic time for foam ripening decreases as the foam liquid fraction decreases [11].

\section{B. Foam invasion regime}

Now we consider the value for the liquid fraction of invading foams. As presented in Fig. 3, $\varepsilon_{t}$ is always larger than $\varepsilon_{\infty}$, indicating that additional liquid enters the pore with each gas bubble. As a gas bubble is sucked into the pore, Plateau borders that initially form the liquid network around the bubble disappear and the corresponding amount of liquid, $V_{\text {sur }}$, is made available at the pore's opening and can be sucked in the pore after the passage of the gas bubble. Whereas in the bulk of the foam, this amount of liquid is shared among three contacting bubbles, $\varepsilon_{\infty}=V_{\text {sur }} / 3 V_{b}$ (where $V_{b}$ is the gas volume of a bubble), the results in Fig. 3 suggest that the whole liquid contained in the surrounding network enters the pore with the bubble. $\varepsilon_{t}$ is thus expected to evolve as $V_{\text {sur }} / V_{b}$, that is, $\varepsilon_{t}=3 \varepsilon_{\infty}$. This behavior compares well with experimental data for $\varepsilon_{\infty}>0.02$ (see Fig. 3), but it appears to underestimate $\varepsilon_{t}$ at low liquid fractions. Obviously, this indicates that the local mechanism for foam invasion is more complex than the simple picture described here. In particular, we have considered that the foam could flow towards the pore's opening in order to fill the volume corresponding to the sucked-in bubble. However, as explained in the previous section, surrounding bubbles can support some stress without flowing. In other words, as a gas bubble is sucked into the pore, surrounding bubbles can partially support the pressure exerted by bubbles from the bulk, allowing the interstitial liquid volume to increase before rearranging. This behavior induces additional liquid to be drained towards the pore's opening, facilitated by the continuous supply of liquid due to gravity drainage. This mechanism can be described using the yield stress of the foam $\sigma_{y}$, or equivalently the yield strain $\Gamma_{y}$, which gives the maximum dimensionless deformation that foam can resist without flowing. This macroscopic property originates from the mechanical behavior of contacting bubbles and is therefore expected to be defined at the scale of a few bubbles around the pore's opening. Note that $\Gamma_{y}$ depends on the liquid fraction and can be 
approximated by $\Gamma_{y} \approx 0.36-\varepsilon_{\infty}$ [12]. The amount of liquid made available by this mechanism is thus of the order $\left(R \Gamma_{y}\right)^{3}$, and the corresponding additional liquid fraction for foam invasion is $\varepsilon_{t}^{+} \approx\left(0.36-\varepsilon_{\infty}\right)^{3} /(4 \pi / 3)$. The liquid fraction $\varepsilon_{t}=$ $3 \varepsilon_{\infty}+b \varepsilon_{t}^{+}$is plotted in Fig. 3 (using $b=1.5$ ) and shows better agreement with experimental data at low liquid fractions. However, the validation of the proposed mechanism would require a dedicated experimental setup, enabling the measurement of the displacement field in the foam during pore invasion.

Whereas the bubble volume was measured to be conserved during invasion of large pores, fractionation was observed with the smallest pore size and largest bubble size. Figure 5 revealed that local bubble rearrangements are responsible for fractionation during invasion. Indeed, the sucked-in bubble experiences contact forces from surrounding bubbles, but the latter are not constant, and instead fluctuate. This is because the foam flow towards the pore's opening proceeds in a succession of local bubble rearrangements. As a consequence, a deviatoric stress appears and the mechanical equilibrium of the sucked-in bubble can be broken. The sucked-in bubble can be disconnected from the pore if its attachment force $F_{t}$ is too small compared to the deviatoric force. A simple expression for $F_{t}$ reads: $F_{t} \approx 2 \pi \gamma r_{t}$. The maximum disconnecting force experienced by the sucked-in bubble corresponds to the situation where a bubble contact is lost whereas a contact is maintained in the opposite direction. In this situation, the detachment force can be modeled by $F_{d} \approx 4 \pi \gamma \delta$, where $\delta$ is the distance over which the bubble is flattened at contact [13]. In the limit of a vanishing liquid fraction, $\delta \approx 0.095 R$. For liquid fractions corresponding to our experiment, we use $\delta \approx$ $0.08 R$ [13]. Balancing $F_{t}$ and $F_{d}$ provides the criterion for fractionation: $R / r_{t} \approx 1 / 0.16 \approx 6$. This value is consistent with the observed transition between nonfractionated and fractionated bubbles.

\section{Gas invasion regime}

Although foam is stable within static conditions, film rupture events are observed under shear conditions imposed by the infusion process, leading to the observed gas invasion regime. Figure 4 indicates that the foam invasion regime is limited by the foam liquid fraction. This behavior can be compared with the recent experiment performed by Biance et al. [9], when triggering bubble rearrangements in foam. Transitions observed between foam invasion and gas invasion in our experiment when $\varepsilon_{\infty}$ is varied coincide with those measured for foam stability and foam collapse in [9]. Consequently, it can be inferred that foam invasion is limited mainly by the intrinsic stability of the foam, rather than specific rupture phenomena occurring at the pore's opening.

This behavior can be understood as follows: When foam is pumped, foam flow proceeds in numerous bubbles rearrangements (the so-called T1 events). During a T1 event, a contact is lost between two bubbles, and a new one is formed between two other bubbles, involving the rapid creation of a new foam film. For the foaming solution used in this study, the typical velocity for the film formation process was measured to be in the range $[9,15,16] u_{\text {film }} \approx 0.2-0.4 \mathrm{~m} \mathrm{~s}^{-1}$. The transient thickness of this new film has been shown to be larger by far than the thickness of other (static) films [9], due to the significant dynamical effects generally observed during the formation of soap films [14]. The volume of liquid transiently involved in the new film has to be present initially in the surrounding Plateau borders, resulting in the existence of a well-defined critical liquid fraction, below which the foam is not stable under pumping conditions. From [9], the critical liquid fraction becomes $\varepsilon_{\text {crit }} \approx \sqrt{2}\left(\eta u_{\text {film }} / \gamma\right)^{4 / 3}$. For the foaming solution used in the present study, one can estimate the theoretical value for $\varepsilon_{\text {crit }}$ to be within the range $0.001-0.003$, which is in reasonable agreement with the measured value (Fig. 4).

\section{CONCLUSION}

We have studied the behavior of a foam forced to pass through a single pore with an aperture smaller than the bubble size. We have observed the formation of a bubble cluster at the pore's opening, and we have shown that the latter can prevent the foam invasion if the invasion flow rate is below a given value $q^{*}$, which depends on the bubble size, the pore aperture, and the foam liquid fraction. A simple model accounts for the observed behavior. Above $q^{*}$, foams invade the pore. The liquid content in the invading foam was measured to be three times larger than the initial one. Finally, for foam liquid fractions below a critical value $\varepsilon_{\text {crit }}$, only gas enters the pore. This has been shown to be related to the intrinsic stability of liquid foams under shear.
[1] R. Höhler and S. Cohen-Addad, J. Phys.: Condens. Matter 17, R1041 (2005).

[2] W. R. Rossen, in Foams in Porous Media, edited by J. F. Sadoc and N. Rivier (Kluwer Academic, Dordrecht, 1999).

[3] M. Chen, Y. C. Yortos, and W. R. Rossen, Colloids Surf., A 256, 181 (2005).

[4] M. Asipauskas et al., Granular Matter 5, 71 (2003).

[5] B. Dollet, J. Rheol. 54, 741 (2010)

[6] S. A. Jones et al., Colloids Surf. A (2010), doi:10.1016j.colsurfa.2010.11.054.

[7] Y. Bertho, C. Becco, and N. Vandewalle, Phys. Rev. E 73, 056309 (2006).

[8] E. Lorenceau et al., Eur. Phys. J. E: Soft Matter Biol. Phys. 28, 293 (2009); F. Rouyer et al., Phys. Fluids 22, 043302 (2010).
[9] A.-L. Biance, A. Delbos, and O. Pitois, Phys. Rev. Lett. 106, 068301 (2011).

[10] D. Weaire and S. Hutzler, The Physics of Foams (Clarendon Press, Oxford, 1999); S. A. Koehler et al., 16, 6327 (2000).

[11] S. Hilgenfeldt, S. A. Koehler, and H. A. Stone, Phys. Rev. Lett. 86, 4704 (2001).

[12] A. Saint-Jalmes and D. J. Durian, J. Rheol. 43, 1411 (1999).

[13] M.-D. Lacasse, G. S. Grest, and D. Levine, Phys. Rev. E 54, 5436 (1996).

[14] M. Durand and H. A. Stone, Phys. Rev. Lett. 97, 226101 (2006).

[15] A.-L. Biance, S. Cohen-Addad, and R. Hohler, Soft Matter 5, 4672 (2009).

[16] E. A. Van Nierop, B. Scheid, and H. A. Stone, J. Fluid Mech. 602, 119 (2008). 